Rechtsmedizin $2017 \cdot 27: 405-406$

DOI 10.1007/s00194-017-0197-3

Online publiziert: 31. August 2017

C) Springer Medizin Verlag GmbH 2017

CrossMark

\section{K. Püschel ${ }^{1} \cdot$ A. Heinemann ${ }^{1}$ - S. Grabherr ${ }^{2}$}

${ }^{1}$ Institut für Rechtsmedizin, Universitätsklinikum Hamburg-Eppendorf, Hamburg, Deutschland

${ }_{2}^{2}$ Centre Universitaire Romand- Médecine légale, Lausanne-Genf (CURML), Lausanne, Schweiz

\title{
Mehr Kontraste in der postmortalen Diagnostik
}

\author{
Postmortale Angiographie/ \\ postmortale CT-Angiographie/ \\ postmortale MRT-Angiographie
}

Erstmals haben wir in der Zeitschrift Rechtsmedizin vor einem Jahrzehnt die postmortale Bildgebung schwerpunktmäßig abgehandelt. Die Überschrift des Editorials lautete seinerzeit: „Schöne Neue Welt von Virtopsy ${ }^{\circledR}$, Autopsy Imaging, Radiosektion und Nekroradiologie“.

Unter dem Oberbegriff der postmortalen bildgebenden Untersuchungen durch Angiographie nehmen wir diese Thematik jetzt erneut auf, nachdem an mehreren rechtsmedizinischen Instituten verschiedene angiographische Techniken evaluiert worden sind. $\mathrm{Zu}$ dieser Untersuchungstechnik gibt es inzwischen eine Reihe von Publikationen, z. T. auch in klinischen Fachzeitschriften. S. Grabherr, J. M. Grimm und A. Heinemann haben einen ersten Atlas herausgegeben [1].

Die postmortale computertomographische Angiographie (PMCTA) ist breit einsetzbar, und zwar sowohl im Zusammenhang mit speziellen rechtsmedizinischen Fragestellungen als auch im interdisziplinären Kontext, bei klinischen $\mathrm{Pa}$ tienten und in Verbindung mit klinischen Sektionen der Pathologen. - In diesem Heft wird dies insbesondere durch die Arbeiten von Vogel et al. sowie Wozniak et al. dargestellt. Vogel et al. zeigen diverse Komplikationen mit Herzverletzungen in der Notfallmedizin: Die Arbeitsgruppe aus Krakau präsentiert 4 Fälle mit Aneurysmaruptur (in unterschiedlicher Lokalisation) sowie eine Herzbeuteltamponade bei Myokardinfarkt.
Dedouit et al. erweiterten den Einsatz der postmortalen Angiographie durch die Verbindung mit einer Magnetresonanztomographie (PMMRTA). Diese Autoren untersuchten mit dieser Technik ein Polytrauma (Sturz aus großer Höhe) sowie einen plötzlichen Tod aus innerer Ursache (mit Aortendissektion). Mit weiteren technischen Modulen werden auch zusätzliche Indikationsstellungen eröffnet.

Heller et al. zeigen die erweiterten diagnostischen Möglichkeiten beim Polytrauma auf. Wittig et al. demonstrieren, wie während der Kontrastmittelinfusion auch der Perfusionsdruck von komprimierten Halsvenen gemessen werden kann, und wie die Darstellung der arteriellen und der venösen Durchblutungsverhältnisse sowie die Pathophysiologie bei der Entstehung von Stauungsblutungen verifiziert werden können.

Eine Zwischenfazit ist möglich: Die postmortale Angiographie hat bereits heute eindeutig ihren festen Platz im Spektrum der Weiterentwicklung bei der postmortalen Bildgebung in der Rechtsmedizin - und damit selbstredend auch in der klinischen Pathologie.

Kritische Hinweise sind zu berücksichtigen: Die Akzeptanz für derartige der Obduktion vorgeschaltete Untersuchungstechniken bei den Obduzenten wird dadurch beeinträchtigt, dass diverse gewebliche Veränderungen durch die Kontrastmittelausbreitung unvermeidlich sind (dies gilt für lipo- und für hydrophile Substanzen). Sowohl die optischen Eigenschaften des Gewebes als auch das „Gewebegefühl“ verändern sich nach vorangehender Kontrastmittelapplikation. Mit Artefakten ist auch in der histologischen Untersuchung $\mathrm{zu}$ rechnen, insbesondere im Hinblick auf Gefäßinhaltsveränderungen, ggf. bei Fettembolien. Unbedingt muss Vorsorge getroffen werden bezüglich chemisch-toxikologischer Untersuchungen. Beispielsweise gehört es zur Routine, vor einer postmortalen Angiographie zumindest Blutproben $\mathrm{zu}$ asservieren (sowohl Herz- als auch peripheres Venenblut) - Standards hierzu entwickeln sich jedoch gerade erst.

Hinzuweisen ist darauf, dass sich in diesem Heft nicht das gesamte Spektrum der diversen angiographischen Untersuchungsmethoden darstellen lässt. Vorzugsweise wird die multiphasische postmortale Angiographie mit einem bestimmten ölhaltigen Kontrastmittel beschrieben. Es sind bereits eine Reihe weiterer technisch etwas anders gelöster Fragestellungen mit anderen Methoden zur Angiographie beschrieben: Zum Beispiel mit anderen lipo- und auch hydrophilen Substanzen, einem anderen Perfusionssystem und geänderten $\mathrm{Ab}$ laufszenarien. Dazu gehört z. B. ebenso die selektive Angiographie für bestimmte ausgewählte Gefäßprovinzen (z. B. die isolierte postmortale Koronarangiographie über ein Kathetersystem, das vor die Koronarostien platziert und durch einen Ballon im Lumen der Aorta blockiert 
wird). Gut bekannt sind z. B. auch Exsitu-Untersuchungstechniken.

\section{》) Visionäres Ziel im modernen rechtsmedizinischen Institut ist die "Untersuchungs- und Bildgebungsstraße"}

Wenn all diese Untersuchungsmöglichkeiten (postmortales CT, MRT, Angiographie usw., z. B. auch die Endoskopie), in Zukunft in der Rechtsmedizin (und in der Pathologie) eingesetzt werden, dann ergeben sich hierdurch komplexe Wechselbeziehungen mit anderen fachärztlichen Disziplinen, insbesondere mit der Radiologie. Zusätzliches Spezialwissen ist erforderlich, ebenso wie eine sehr enge Kooperation. Es entstehen die Fragen, inwieweit (forensisch ausgerichtete) Radiologie verpflichtend in den Weiterbildungskatalog der Rechtsmedizin aufgenommen wird, und wie klinische Radiologen vermehrt für rechtsmedizinisches Basiswissen gewonnen werden können, um postmortale Befunde mit den verschiedenen Bildgebungstechniken zu interpretieren. Daraus entwickeln sich dann Wortspielereien wie „Nekroradiologie“, „Forensische Radiologie“ oder auch „Forensische Radiopathologie“. Diese Überlegungen lassen sich noch sehr weitgehend fortsetzen, und es bleibt abzuwarten, wie dies in Zukunft praktisch umgesetzt wird. - Das visionäre Ziel in einem modernen rechtsmedizinischen Institut ist eine „Untersuchungsund Bildgebungsstraße", wie sie in einzelnen Instituten schon in Ansätzen realisiert ist: untereinander verschaltete Räume mit Spezialausstattung, verbunden mit einem automatisierten Leitsystem für den Leichnam. Beginnend mit der Spurensicherung von Kleidung und Körperoberfläche führt die „Untersuchungs- und Bildgebungsstraße“ über die dreidimensionale, maßstabsgerechte fotografische und gescannte Dokumentation der gesamten Körperoberfläche hin zu CT, MRT, Angiographie, Endoskopie und schließlich zu vom Bildschirm aus gesteuerten autoptischen/ bioptischen Prozeduren mithilfe von Robotersystemen. Dies alles ferngesteu- ert von einer Technikzentrale mit allen Möglichkeiten der Bildmischung und der Fernübertragung von Daten, sodass der Untersuchungsort des Leichnams telemedizinisch mit den Experten in einer Bildauswertungszentrale eines zentralen Instituts verbunden ist.

All diese Optionen existieren schon heute in der Animationstechnik, der klinischen und punktuell der rechtsmedizinischen Routine. Man muss nur die Initiative ergreifen und die Ressourcen bereitstellen, um diese faszinierende Vorstellung umzusetzen.
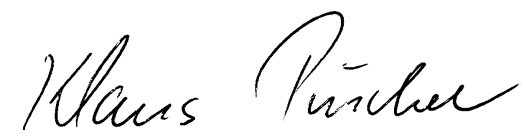

Klaus Püschel

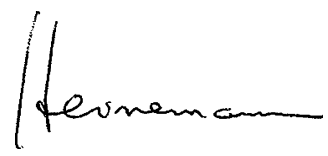

Axel Heinemann

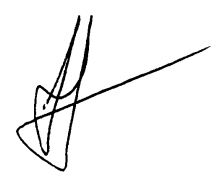

Silke Grabherr

\section{Korrespondenzadresse}

Prof. Dr. K. Püschel
Institut für Rechtsmedizin,
Universitätsklinikum
Hamburg-Eppendorf
Butenfeld 34, 22529 Ham-
burg, Deutschland
pueschel@uke.de

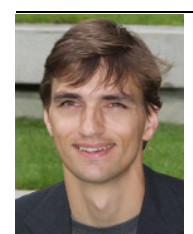

Dr. A. Heinemann

Institut für Rechtsmedizin, Universitätsklinikum Hamburg-Eppendorf Butenfeld 34, 22529 Hamburg, Deutschland heinemann@uke.de

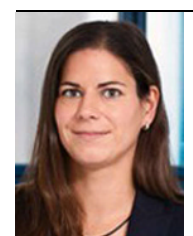

Prof. Dr. S. Grabherr

Centre Universitaire RomandMédecine légale, LausanneGenf (CURML)

Chemin de la Vulliette 4, 1000 Lausanne, Schweiz Silke.grabherr@chuv.ch
Interessenkonflikt. K. Püschel, A. Heinemann und S. Grabherr geben an, dass kein Interessenkonflikt besteht.

\section{Literatur}

1. Grabherr S (2016) Atlas of Postmortem Angiography. Springer, Cham, Heidelberg, New York, Dordrecht, London 